\title{
A Smart Assistance Solution for Remotely Monitoring the Orthopaedic Rehabilitation Process Using Wearable Technology: re.flex System
}

\author{
Marilena IANCULESCU ${ }^{1 *}$, Bogdan ANDREI ${ }^{2}$, Adriana ALEXANDRU ${ }^{1}$ \\ ${ }^{1}$ National Institute for Research and Development in Informatics, \\ 8-10 Averescu Avenue, Bucharest, 011455, Romania \\ marilena.ianculescu@ici.ro (*Corresponding author), adriana.alexandru@ici.ro \\ ${ }^{2}$ Cetrokinetic, 18, Mircea Eliade, Bucharest, Romania \\ dr.andrei.bogdan@centrokinetic.ro
}

\begin{abstract}
Physical therapy helps patients during their rehabilitation programme after orthopaedic post-operative interventions or in case of musculoskeletal injuries. For a full recovery and a minimisation of the risk of complications, the prescribed in-clinical therapy sessions must be supplemented by independent home training. The purpose of this paper is to present the emerging technologies used for monitoring ortophedic movements and to introduce an innovative telerehabilitation-as-aservice solution, called re.flex, which consists of two IMU (Inertial Measurement Unit) motion tracking sensors and a mobile application The therapist makes use of standardised digitised physical exercises that are carefully chosen so as to create, by selection, a custom designed training program that will meet the needs of each patient. The feedback provided by the sensors enables the therapist to a) monitor the patients' compliance with the training program in a transparent manner, b) keep track of the documentation related to the recovery progress through data-collection during in-clinical and in-home exercise-based rehabilitation c) customize the training program. The real-time 3D smartphone animations and the direct visual, audio and haptic feedback from the sensors assist patients in performing the rehabilitation exercises assigned to them. The abovementioned application allows the patients to visualise their injured limb on their smartphone or tablet in an innovative 3D $360^{\circ}$ manner, thereby reducing their fear of making mistakes during the recovery process and offering them constant security. The study evaluates the effects of this kind of approach for a group of 30 participants and the results recorded are in favour of the patients recovering with re.flex.
\end{abstract}

Keywords: Othopaedic physiotherapy, Telerehabilitation, IMU sensors, Motion tracking sensors, Orthopaedic musculoskeletal assessment platform for post surgery care.

\section{Introduction}

All over the world, there is an important constraint from governments to improve efficiency and productivity in medical activities, to empower patients to manage their own health and care and to improve health results (Alexandru \& Ianculescu, 2017).

Physiotherapy is a growing field of medicine, due to the necessity of adequate recovery after injuries, but also to the sedentary lifestyle of people. Annually, there is a growing number of knee replacements caused by the rise of life expectancy and by the growing rate of global obesity.

Information and Communication Technologies (ICT) facilitate home treatment in musculoskeletal physiotherapy by offering various solutions for telerehabilitation and home treatment, as part of eHealth approaches. Mobile applications with lots of video exercises centered on patient and his education and customized in protocols based on age and gravity of injuries may prove instrumental in improving the health of patients. By using video exercises, the patients can get accustomed with the rehabilitation treatment based on a comprehensive video support.
The patients and the providers of post-operatory orthopaedic therapy and rehabilitation activities can enhance the recovery programs as well as the healthcare results, Thus, patients can take an active part in their own healthcare recovery by adopting home-based solutions as alternative solutions for in-clinic post-operatory rehabilitation. The short and medium term results meet the patients' needs and are similar to those recorded in clinics. As a result, the reduction in treatment expenses and in the number of hospitalization days has a positive impact on costs and productivity. The patients are no longer overloaded by transportation and accommodation costs, or by the waiting times. In addition, patients' complications due to hospitalacquired infections are minimized.

The inconvenient of this approach is the necessity of constant human supervision, because the therapist is aware of what the patient is doing. This aspect can be overcome by employing new technologies in rehabilitation based on the usage of wearables in health domain applications. Wearable sensors have a small size, can be worn $24 / 7$ during the recovery period, are able of estimating a body position and overall compliance 
with the physical exercises designed for bending and extension of the knee.

Remote patient assessment platforms for orthopaedic care provide physiotherapy possibilities for patients' total rehabilitation at home under self-direction. The clinicians manage remotely the recovery process and obtain all the data that enable them to evaluate the recovery progress of each patient. They convey their knowledge and offer their assistance and encouragement by real-time video sessions. Remote physiotherapy is a step further to better solutions based on IMU sensors, which are "electronic devices that measure and report orientation, velocity, and gravitational forces through the use of accelerometers and gyroscopes and often magnetometers" (Olson \& Redkar, 2018). The data from these sensors are collected online. The incorporated IMU sensors on mobile applications for physiotherapy provide user-friendly video exercises and are used both for training the patients to understand the exercises, and for facilitating the real-time data collection on patients' home activity. By using these data, the physiotherapist can perform a health assessment with a view to deciding on the appropriateness of changing the rehabilitation exercise plan for a certain patient.

re.flex is a system that helps patients, physiotherapists and clinical managers who are involved in the recovery process after injuries by making the medical and managerial staff more efficient and contributing to an easier recovery for patients. re.flex is focused on knee and hip physiotherapy, being the most advanced orthopaedic tele-rehabilitation solution (Radicle, 2018).

This paper aims to present the results of a randomized controlled trial of an innovative system, re.flex, that highlights how an IMU-based remote patient monitoring solution can improve the orthopaedic rehabilitation.

The rest of the paper is organized as follows: Section 2 presents some of the main emerging technologies used for monitoring the orthopaedic movements and current applications based on them. Section 3 briefly introduces the reffex system and describes the first orthopaedic telerehabilitation study with sensors in Eastern Europe. Section 4 marks out how the ongoing RO-SmartAgeing project can be improved with the help of the technology developed by re.flex. Some concluding insights are presented in the final section.

\section{Related Works on Applications Based on Emerging Technologies Used for Monitoring Orthopaedic Movements}

New emerging technologies represent a compulsory path for developing comprehensive and customized solutions, including the remote monitoring and rehabilitation. They feature an increasingly high capacity to continuously track the therapy and its results, to better engage the patient in the recovery process with minimal human assistance, and to enhance the efficiency of the orthopaedic activity, including its cost-effectiveness.

Some of the most important applications used for monitoring orthopaedic movements are presented below:

\subsection{Video Applications}

Video applications are easily accessible and usually inexpensive tools that aim to sustain the physical therapy performed at home by the patients, as a part of the medical recovery plan. They may include flexible, personalized exercise programs, constant guidance, video demos or instructive health information.

\subsubsection{Physitrack}

Physitrack apps allow access to more than 4.200 explicitly explained clinical home exercise videos, new 3D content, remote healthcare features and an outcome analysis available on patients' smartphones or computers. The targeted domains are physical rehabilitation and prevention for Orthopaedics, Neurology, Pediatrics, Cardiology and Women's Health (see Figure 1) (Physitrack, 2019).

\subsubsection{Kaia Health}

Kaia Health applications represent AI-powered applications that include physiotherapeutic and psychological exercises.

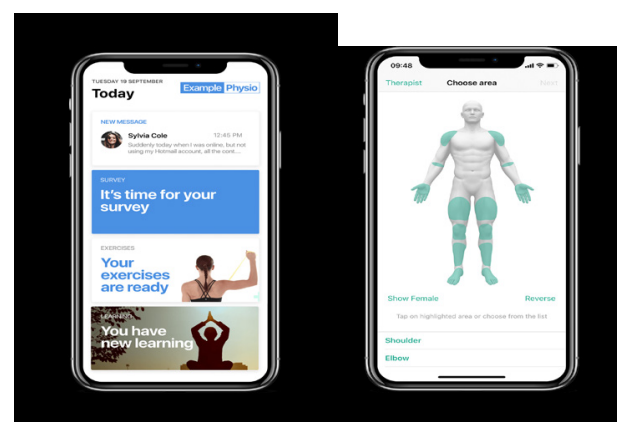

Figure 1. "Patient onboarding \& triage" Physitrack apps (Physitrack, 2019) 
They are based on a therapy algorithm that allows a customized content to be associated with the patient at the proper time, whereas in-app tracking features enable the patient to control his progress in accordance with different metrics, such as pain levels. An AI-powered motion tracking technology screens the person's motions by means of an iPhone camera (see Figure 2).

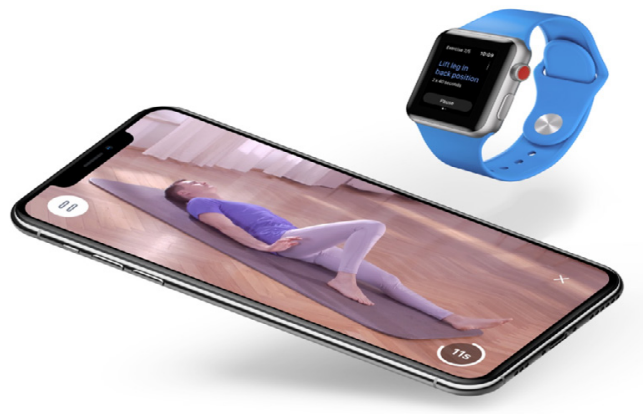

Figure 2. "Back Pain Exercises" Kaia app (Kaia Health, 2019)

Based on machine-learning models, Kaia's app is also able to inform the patient in natural language if the exercises are properly done or, if not, what direction has to be taken. (Kaia Health, 2019).

Some of the limitations of video applications are: certain recovery outcomes (such as knee and hip flexion or extension) that cannot be measured; the provided video exercise databases aren't automatically compared with patient's movements; as the data are self-reported by the patients, they can be accidentally or deliberately misreported, which has direct consequences on establishing the patient's real adherence to the exercise plan and his real recovery progress.

\subsection{Human Motion Tracking Systems}

Human motion tracking systems use a human body model with associated parameters which are assessed based on the available data while the body modifies his posture in a defined space (Vera-Perez et al., 2014). An accurate tracking can be done with wearable sensors or with high-speed cameras. The provided motion analysis makes them appropriate for orthopaedic rehabilitation.

Human motion tracking systems are grouped in two categories: computer vision-based and wearable sensor-based systems (Xu et al., 2019). The latter are appropriate for dynamic functions and continuous monitoring, since they provide an accurate and quantitative analysis of the available data, while involving a low-cost usage.

\subsubsection{Kinect}

Kinect is a Microsoft motion device that is able to track up to six bodies inside its associated view area. The device contains a depth sensor that can set up full-body 3D motion capture features. Based on an embedded skeleton algorithm, up to 25 joints for every identified body are provided in 3D (Beshara et al., 2016; Naeemabadi et al., 2018). A number of research studies were carried out in order to evaluate the precision and sustainability of Microsoft Kinect sensors for static postures (Xu \& McGorry, 2015; Darby et al., 2016), gait (Mentiplay et al., 2015; Auvinet et al., 2017; Eltoukhy et al., 2017), body sway (Yeung et al., 2014), and joints angles for certain physical activities (Anton et al., 2016; Woolford, 2015; Huber et al., 2015).

Among the limitations of the Microsoft Kinect device, the most distinctive are: the necessity to eliminate from the associated view area all the objects that might cover the body, the incapacity of tracking the exercises done in a lying position, (Naeemabadi et al., 2018), the high production cost (over $1.600 \$$ ), the fact that it cannot be customized according to the patient's needs, the inability of detecting supine positions without extra hardware sensors.

\subsection{Avatars Used for Rehabilitation}

Animated avatars have a well-defined potential for supporting the patient's engagement in-home rehabilitation phases due to the latest developments in motion tracking devices or machine learning. The capacity of an avatar to establish a direct communication with the patient enables the emergence of a better customized environment, which is perceived as an extension of the patient in terms of prevention, data recording applications and recovery (Shaked, 2017).

\subsubsection{Virtual Exercise Rehabilitation Assistant (VERA)}

VER $\boldsymbol{A}$ is an example of a digital platform that uses such an animated avatar to provide a wide range of guided physical exercises which are appropriate for therapy and rehabilitation in a nonclinical environment, together with tools which can ensure a remote monitoring of the patient. VERA comprises 3D motion-capture technology, it can measure 25 joints and limbs in motion at 
a rate of 30 times per second, it records exercise performances providing audio-visual feedback (see Figure 3). It is strongly oriented towards the patient not only through the VERAHome system, but also through the VERAClinic one that supports the physicians in monitoring and defining customized therapy plans for their patients (Reflexion Health, 2019).
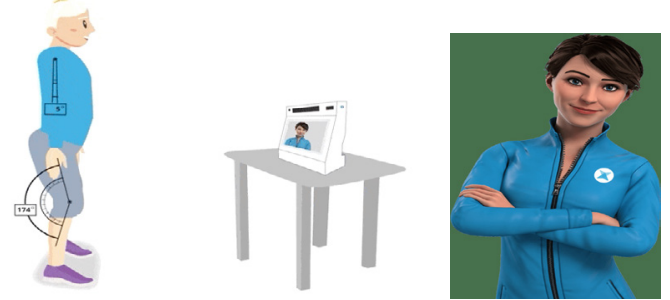

Figure 3. VERAHome system (Reflexion Health, 2019)

\subsection{Inertial Measurement Unit (IMU) Sensors}

An IMU sensor estimates angular rate, force or magnetic field. This sensor is frequently associated with a sensor fusion software that puts together data from several sensors for measuring limb orientation and direction. The devices based on IMU sensors are affordable and appropriate for precise, non-invasive and portable motion tracking (Filippeschi et al., 2017).

\subsubsection{Systems Using One IMU Sensor}

The systems using one IMU sensor can be efficient in analyzing the exercise technique (O'Reilly et al., 2017). Advanced mathematics allow a single sensor to be used with a plain strap attachment, which counterbalaces minor position impreciseness.

\subsubsection{BPMpathway}

BPMpathway is a cost-effective remote patient assessment platform that supports the orthopaedic patients and their clinicians by elaborating a customized post-surgery in-home rehabilitation programme. An IMU sensor worn on the limb sends data to the BPMpathway app on the patient's tablet, which displays the results on a live animated avatar. The data is compared in real-time and displayed while it is automatically sent through cloud computing to the personal physician for evaluation (see Figure 4) (BPMpathway, 2019).

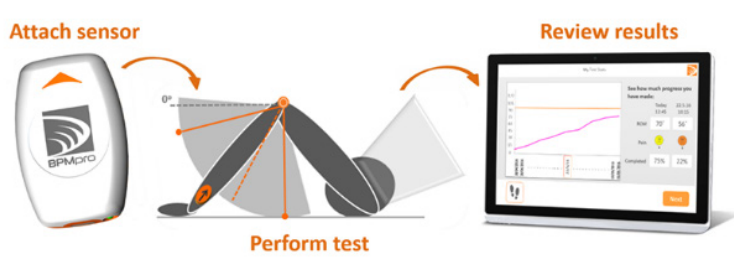

Figure 4. BPMpathway functioning (BPMpathway, 2019)

\subsubsection{2 inter ACTION}

interACTION is a remote rehabilitation management portable system that is based on two IMU sensors, each composed of a tri-axial accelerometer, gyroscope, and magnetometer sensors (see Figure 5A), an interactive app and a web-based physician portal.

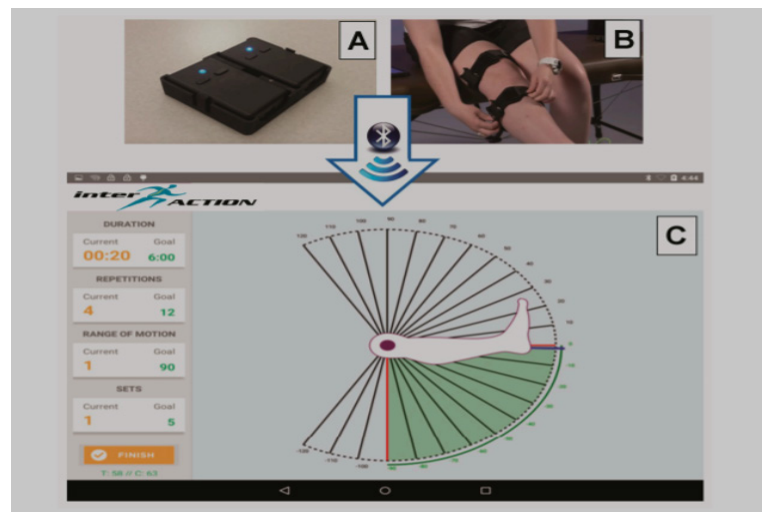

Figure 5. interACTION portable system functioning (Bell et al., 2019)

The two sensors (see Figure 5B) provide a realtime visual feedback that allows the patient to self-monitor and adapt the exercises, and the physician to remotely assess the patient's adherence to exercises and his recovery (see Figure 5C) (Bell et al., 2019).

\subsubsection{SWORD Health}

SWORD Health is a low-cost kinematic biofeedback tool based on three IMU sensors, AI and cloud technology that targets in-home physiotherapy. An AI physical therapist establishes personalized exercises, and a motion tracker system digitizes the patient's movements and delivers a real-time feedback through a mobile app (see Figure 6). The patient is better involved in the continuous improvement of the rehabilitation 
under the strict remote supervision of a human clinical team (SWORD Health, 2019).
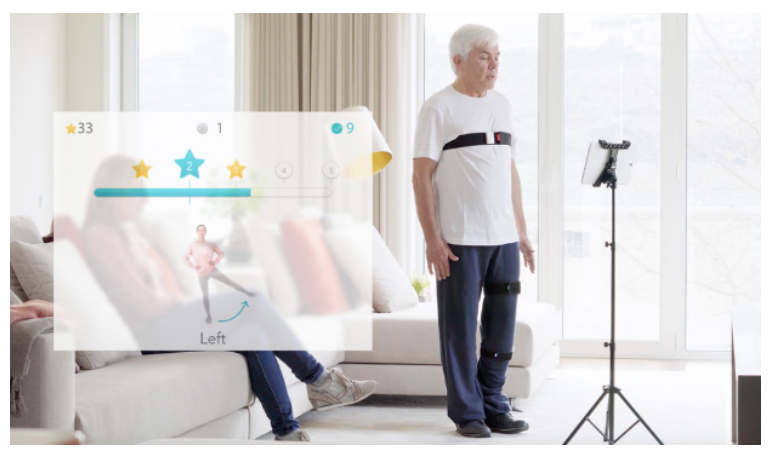

Figure 6. Digital Therapist from SWORD Health (SWORD Health, 2019)

\section{Case Study: re.flex System}

\subsection{Brief Presentation}

re.flex is an innovative system that aims to monitor the patients' musculoskeletal physiotherapy for the knee. It is appropriate for use both in clinics and at home as a smart rehabilitation assistant and allows open access to physical rehabilitation.

\subsection{Comparative Analysis}

re.flex offers $3 \mathrm{D}$ detection and $3 \mathrm{D}$ visualization facilities. So, knee and hip flexion and extension can be measured and supine positions can be detected. Enough video exercises are available for structuring a post operative rehabilitation protocol. The tracking of the exercises and protocols is possible. re.flex is suited for patients' usage and can be customized based on their needs.

\section{3 re.flex Architecture}

The architecture of re.flex includes:

- Two motion IMU sensors that collect data related to twelve movement metrics (orientation, velocity, gravitational forces, etc.) used for analyzing the patient's movements (see Figure 7). So, 90\% of the most usual knee exercises can be performed (re.flex, 2018). The digital data acquired by the IMU sensors is considered to be a useful tool for monitoring patients recovery.

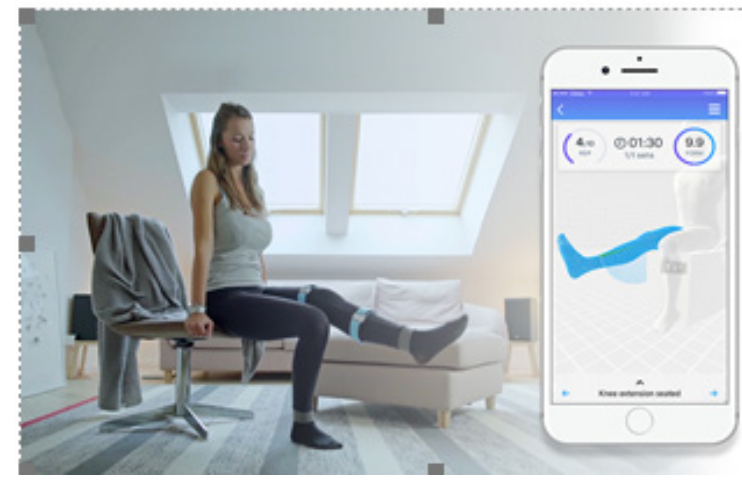

Figure 7. re.flex wearable sensors

The motion sensing devices are mounted above and below the injured joint of a patient.

The hardware sensors contain three axis accelerometers, three axis gyroscopes. Different from other motion tracking implementations, the system does not use a magnetometer as it's very susceptible to interference with iron based objects, which are very common in the physiotherapy clinics and even at home.

The placement of the sensors on the body is very important for other solutions as it influences all the subsequent measurements. This is why other solutions rely on very careful placement of the sensors that can be recognized by their algorithms. From the developers' perspective it's very hard for the patient to accurately place the sensors in the desired position: the desired position might be over the injury, it's not comfortable to wear the sensors in those positions, the patient has different sized limbs than the standard, etc. Another issue is that careful placement means lost time and additional worries for the patient who should concentrate on recovering from the injury. This might affect the adherence to the treatment plan which is not desirable.

This is why re.flex allows the motion sensing devices to be placed randomly above and below the patient's affected joint. The patient doesn't have to fix them in a certain position; the auto-calibration algorithm detects the actual positioning and ensures that the orientations of the sensors are related to the actual physical axis of rotation of the join.

- A mobile application that receives realtime sensor data about patients' activity, received wirelessly from IMU sensors. Using a complex movement algorithm, an innovative, precise 3D - 360-degrees realtime visualization of the injured limb on smartphone is provided (see Figure 7). 
The mobile application is made of two separate interfaces, a physiotherapist interface and a patient interface. These two different interfaces are accessible using the corresponding login parameters; if the user logging-in is a patient, then the patient interface is shown, if the user is a physiotherapist, the physiotherapist interface is shown instead.

The mobile application is customized for the iOS and Android operating systems. For the iOS operating system, the application is made of a Swift coded user interface, a $\mathrm{C}++$ coded movement and calibration algorithm and a Unity coded live render view. For the Android operating system, the application is made of a Java coded user interface, a $\mathrm{C}++$ coded movement and calibration algorithm and a Unity coded live render view.

The mobile application includes as well an Exercise definition module, an exercise scheduling module and a movement analysis algorithm.

The quality of the movements can be appreciated based on the comparison between the healthy leg and the injured one. Physiotherapists can access easy-tounderstand reports about patient adherence, pain, flexion and quality of the movement (Socker, 2018).

The physiotherapist interface shows all the patients that physiotherapist has registered, their progress, the list of scheduled exercises, feedback from the patient and patient assessment module.

The physiotherapist has a database of exercises to choose from or predefined programs that can be assigned to a patient depending on the affection/surgery.

The exercise database contains a list of predefined exercises that the physiotherapist will use as a base for his own exercise programs. Each exercise can be customized within the physiotherapist application so it fits the patient using the system.

The patient interface contains a list of exercises prescribed by the physiotherapist to the patient accessing the web application. This list can be updated by the physiotherapist using the web platform or the mobile application. The mobile application receives data from the motion sensing devices, shows the movement live on the mobile device screen, counts exercise repetitions and shows how correct the movement was done using the thresholds set previously by the physiotherapist.
A medical platform (the web server) where exercises and exercise programs are defined and scheduled for patient. The video exercises and recovery programs creates a link between the patient and the physicians based on an insight full analysis of patient's stored data or a customized monitoring, assessment and immediate feedback. The Web server also acts as a physiotherapist interface to the cloud-based database which stores all the exercise/programs data and the patient data. It allows defining new exercises by setting movement constraints and changing existing exercises. Continuous synchronization with the physiotherapist and patient mobile applications ensures the exercise database is always up to date.

The web server is coded in the Javascript language and has a MySQL database.

The web platform allows exercises and exercise programs to be defined by the physiotherapist or by the clinic. There are standard public programs defined and available for all the clinics and physiotherapists, and private programs that can be seen only by the clinic.

The web platform allows the physiotherapist to see all the exercises done by the patient, the progress of the recovery and to adjust the program scheduled for the patient.

The progress of the recovery can be measured during the first weeks of the recovery by the flexion increase. This is the phase where the patient tries to recover the lost flexion due to the injury. In this phase all progress and exercises are targeted towards the recovery of function for the patient. The phase is assumed to be completed when the patient recovers flexion to the values previous to the injury.

The next phase of recovery is targeted towards strengthening the muscles and recovering to the previous state before the injury. The progress in this phase is measured by the number of exercises done, the increased duration of isometric exercises and the increased weights used for the training.

- A cloud-based database which keeps the physiotherapist, patient and exercise data.

- A two-way communication system, available in the mobile app, and useful when the patients have urgent questions or the physiotherapist needs to intervene.

re.flex cannot replace conventional physiotherapy, but it can help this industry to scale up more easily. Basically, a specialist can treat multiple people at the same time, because the monitoring will be 


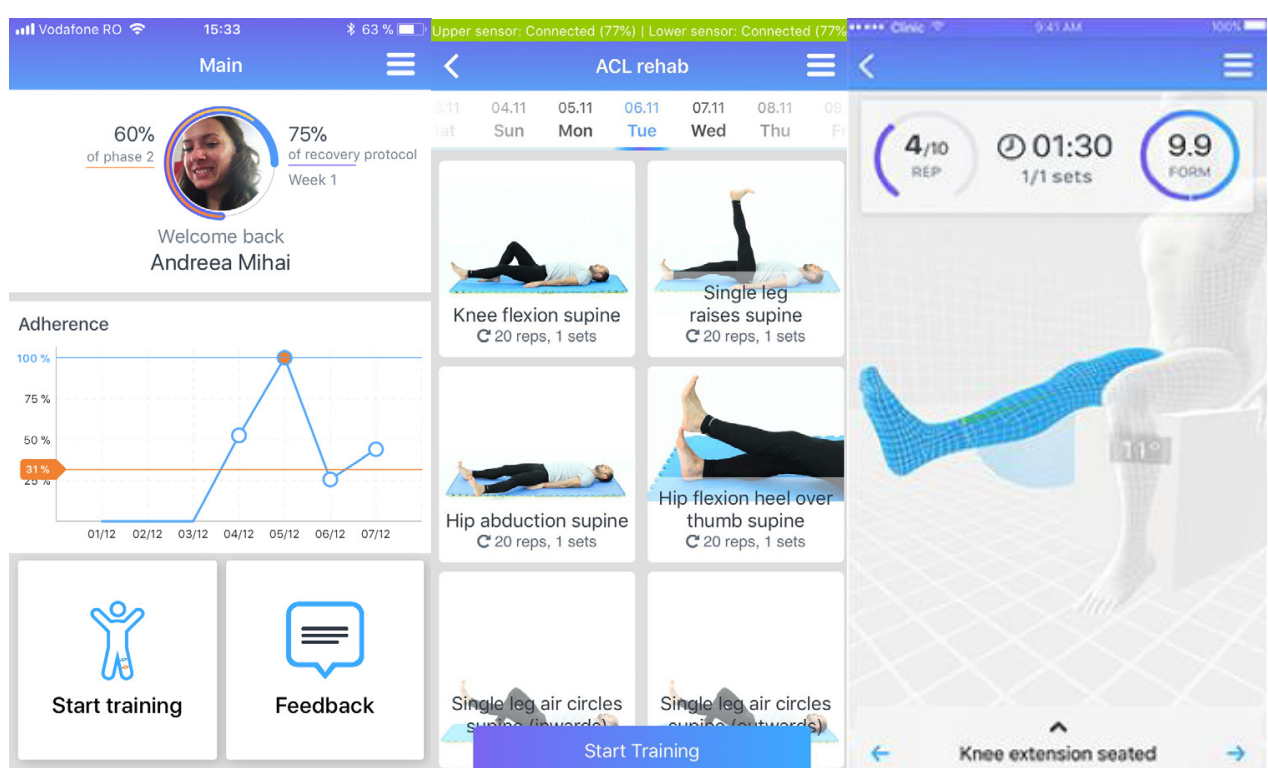

Figure 8. Virtual assistance provided by re.flex (re.flex, 2018)

done by the IMU sensors of the system. re.flex can measure the evolution of a patient's recovery based on the measurements made on that patient when he entered the clinic. If a physiotherapist puts the IMU sensors on the patients, they can also visualize the physical exercises which are performed, receive notifications in the form of vibrations when they do not perform well, and they are also monitored by the system which enables them to correct the execution of the exercises (see Figure 8).

As a digital partner for healthcare providers re.flex develops the IMU sensors and the visualization system (software) for the use of clinics, physiotherapists and hospitals. Patients rent these sensors and take them home, so that they may be able to track their own progress after a certain level of recovery. The rehabilitation protocols developed by re.flex follow the guidelines of the most prestigious universities. Also, can have their own protocols included in the recovery programs or some protocols developed by the head of the physiotherapy. Over time, specialists will be able to create their own programs that have the potential to better utilize the healthcare resources. The system owners and medical managers can see the protocol, if the recommended exercises have been done, how long they will work with the patient, how busy the specialists are and how they work.

\subsection{Experimental Study}

\subsubsection{Data Acquisition}

The first orthopaedic telerehabilitation study with sensors in Eastern Europe started in May 2018, re.flex being coordinated by Dr. Bogdan Andrei, a leading orthopaedic doctor from Floreasca Emergency Hospital and also the owner of Centrokinetic, a leading orthopaedic rehabilitation clinic in Bucharest, Romania. Dr. Bogdan Andrei got involved in re.flex as an orthopaedic advisor.

One of the most common knee injuries is an anterior cruciate ligament (ACL) sprain or tear. 30 patients following an ACL reconstruction were selected to take part in the research, from day two after surgery.

The patients were divided into two equal groups and randomly allocated to either the control group or the intervention group. They received 12 weeks of physical therapy treatment. The former group has been treated in a clinical setting, while the latter received the same treatment at home, while being monitored with re.flex.

The knee flexion was measured 2 days after the ACL reconstruction surgery had been performed and for the intervention group it varied between 30 and 80 degrees.

\subsubsection{Data Analysis}

The outcomes of the recovery for patients following an ACL reconstruction depend on multiple factors: timing and the type of surgery, timing of the recovery, adherence to the recovery plan, total amount of time invested in the recovery and patient's medical state. If one documents the level of importance for each of the abovementioned factors by using technology, this can 
create more advanced recovery protocols and help patients recover better and faster. After the 4 months of treatment with re.flex, the patients' knee flexion was measured again and significant changes have been recorded. All patients were satisfied with their range of motion and compared with the healthy leg, they had achieved full flexion. An increase of $31 \%$ in the adherence to the recovery program was recorded for the patients in the intervention group, that received 16 weeks of physiotherapy through the re.flex system (see Figure 9).

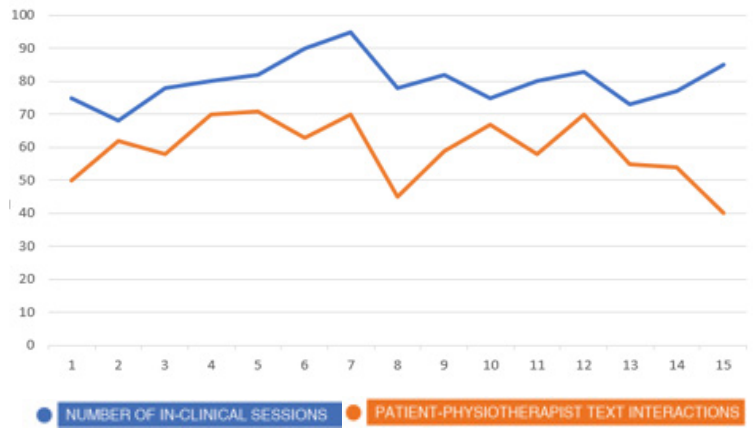

Figure 9. Difference of adherence between the groups

Physiotherapists were able to track the correctness score of home sessions. Another important aspect that has been studied by using the sensors was the correctness score of the home exercises performed. This innovative metric is only available for the sensor solutions that gather and analyze real-time data.

The correctness score offers the physiotherapist a strong hint about how the patient performs the exercises because it takes into consideration if the proper levels of joint constraints' angles are met. By studying the data collected by the sensors, it was noticed that patients were gradually increasing their correctness scores, as they learned the exercises and the correct movements. The outcomes were measured using the Oxford Knee Score. Patients completed the form on the second day after the surgery and at the end of the fourmonth treatment (see Figure 10).

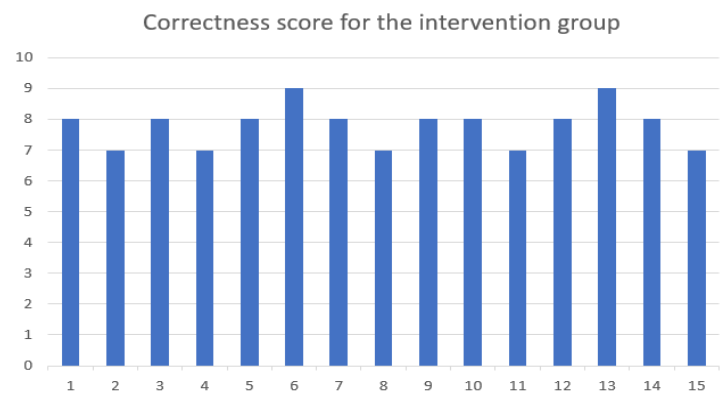

Figure 10. Correctness score for the intervention group

\subsubsection{Results}

An average difference of 1.3 OKS (Oxford Knee Score) points was recorded in favour of the intervention group. Therefore, the patients were satisfied with the results obtained by this approach in terms of the improvement of postoperative quality of life, which lead to a faster reintegration into the social environments.

Patients from the intervention group visited the clinic on average 2 times in the course of the 4-month treatment. During the first visit they've learned how to use the above-mentioned solution while the second, and in few cases, the third visit, was a follow-up visit. It was interesting to analyse the number of interactions that the patients had had with their remote therapist. $90 \%$ of the interactions took place through the application and 8-15 message exchanges were recorded (see Figure 11).

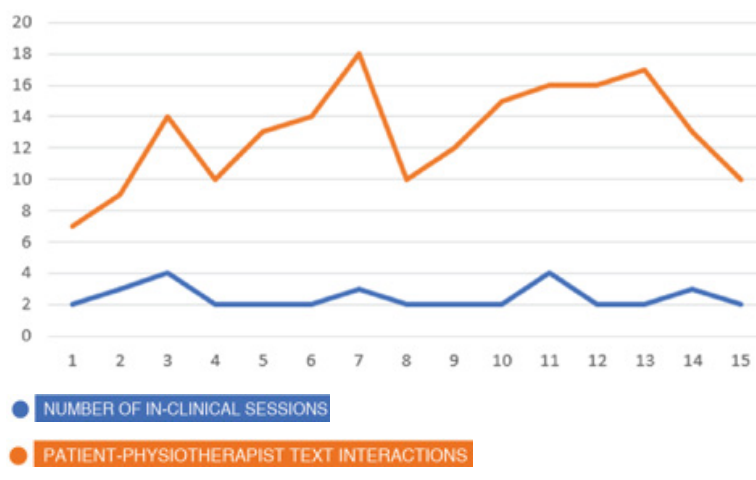

Figure 11. Message exchanged through the app and during hospital visits

This difference can be linked to multiple factors, including openness to technology, a high degree of helplessness, or the quality of the therapeutic alliance and should be studied further.It has been observed that patients used the chat in the re.flex application mostly for finding answers about the pain they have felt during the treatment. However, at the end of the treatment, all patients were satisfied with the recovery and the delivery of the treatment.

The physiotherapists were asked to take part in a 15-item questionnaire structured into: the satisfaction degree regarding the recovery solution, and their level of implication required in order to deliver the treatment to their patients. The recorded satisfaction rate was $94 \%$. From the patient's perspective, satisfaction was also high. The level of patients' satisfaction with the method of treatment and feedback was very good $(90 \%)$.

Both, physiotherapist and patients reveal the necessity of having available similar systems useful in the rehabilitation of other body parts.

https://www.sic.ici.ro 


\section{Potential for Further Developments}

Remote health monitoring of the elderly consists in multivalent procedures based on a technology adapted to the particularities induced by ageing, and performed in non-clinical environments (Ianculescu et al., 2018). The elderly`s activity recognition, with a special focus on motion, which was recorded through appropriate sensors is an added value (Voicu et al., 2019). The particular importance of health data imposes the deployment of innovative tools able to harmonize and interconnect it (Esposito et al., 2017).

"Non-invasive monitoring and health assessment of the elderly in a smart environment (ROSmartAgeing)" is an ongoing project that aims to support an active and independent life of the elderly at home, the prevention and rehabilitation of age-related dysfunctionalities, and a wider access to improved personalized healthcare.

As RO-SmartAgeing is based on IoT, cloud computing, and predictive analysis technologies, integrating some components of re.flex system, it can enlarge its designed functionalities by allowing an early detection of the decline of the elderly patient's locomotor system and identifying the most suitable fall prevention frameworks, gait analysis and management of the lower back pain and other musculoscheletal conditions of the spine. The teams involved in the above-mentioned projects are assessing the opportunity of elaborating an IMU-based algorithm for detecting daily activities that can be associated with a remotely guided personalized physiotherapy.

\section{Conclusions}

This paper highlights the support brought by some emerging technologies for the accurate monitoring of orthopaedic movements, physiotherapy and rehabilitation, highlighting how the best functional outcomes can be obtained in compliance with the patient's adequate involvement.

re.flex is an innovative system with a strong motivational feature for the patients. The smart rehabilitation assistant allows an accurate monitoring and evaluation of the customized exercises. In the near future, besides the current focus on lower limb rehabilitation, re.flex solution will be augmented by means of a lower back 3D support, hand and shoulder exercises and protocols. The potential of extending functionalities of the ongoing RO-SmartAgeing system by integrating the re.flex telerehabilitation-as-a-service solution was identified and is under development. This approach will help the older people to recover their muscle balance ability, to diminish their pain, and to enhance their quality of life.

\section{Acknowledgements}

The authors gratefully acknowledge the contribution of the Romanian Ministry of Research and Innovation for funding the projects RO SmartAgeing - Non-invasive Monitoring System and Health Assessment of the Elderly in a Smart Environment for the period 2019-2022 and "vINCI: Clinically-validated INtegrated Support for Assistive Care and Lifestyle Improvement: the Human Link" for the period 2018-2021.

\section{REFERENCES}

1. Alexandru, A. \& Ianculescu, M. (2017). Enabling assistive technologies to shape the future of the intensive senior-centred care: A case study approach, Studies in Informatics and Control, 26(3), 343-352. DOI: 10.24846/ v26i3y201710

2. Anton, D., Nelson, M., Russell, T., Goñi, A. \& Illarramendi, A. (2016). Validation of a Kinectbased Telerehabilitation System with Total Hip Replacement Patients, Journal of telemedicine and telecare, 22(3), 192-197.

3. Auvinet, E., Multon, F., Manning, V., Meunier, J. \& Cobb, J. P. (2017). Validity and sensitivity of the longitudinal asymmetry index to detect gait asymmetry using Microsoft Kinect data, Gait \& Posture, 51, 162-168.

4. Bell, K. M., Onyeukwu, C., McClincy, M. P., Allen, M., Bechard, L., Mukherjee, A., Hartman, R. A., Smith, C., Lynch, A. D. \& Irrgang, J. J. (2019). Verification of a Portable Motion Tracking System for Remote Management of Physical Rehabilitation of the Knee, Sensors, 19(5), 1021.

5. Beshara, P., Chen, J., Lagadec, P. \& Walsh, W. R. (2016). Test-retest and Intra-rater Reliability of Using Inertial Sensors and its Integration with Microsoft Kinect to Measure Shoulder Range-of-motion. In International Conference on IoT Technologies for HealthCare (pp. 177184). Springer, Cham.

6. BPMpathway (2019). The proven dimension in remote patient assessment. Available at: $<$ https://www.bpmpathway.com/bpmpathwayproduct-description/>. 
7. Darby, J., Sánchez, M. B., Butler, P. B. \& Loram, I. D. (2016). An evaluation of 3D head pose estimation using the Microsoft Kinect v2, Gait \& Posture, 48, 83-88.

8. Eltoukhy, M., Oh, J., Kuenze, C. \& Signorile, J. (2017). Improved kinect-based spatiotemporal and kinematic treadmill gait assessment, Gait \& Posture, 51, 77-83.

9. Esposito, C., Castiglione, A., Tudorica, C. \& Pop, F. (2017). Security and privacy for cloudbased data management in the health network service chain: a microservice approach, IEEE Communications Magazine, 55(9), 102-108.

10. Filippeschi, A., Schmitz, N., Miezal, M., Bleser, G., Ruffaldi, E. \& Stricker, D. (2017). Survey of Motion Tracking Methods Based on Inertial Sensors: A Focus on Upper Limb Human Motion, Sensors, 17(6), 1257.

11. Huber, M. E., Seitz, A. L., Leeser, M. \& Sternad, D. (2015). Validity and reliability of Kinect skeleton for measuring shoulder joint angles: A feasibility study, Physiotherapy, 101(4), 389-393.

12. Ianculescu, M., Alexandru, A. \& Rădulescu C. Z. (2018). Patient-centered innovative monitoring system and smart personalized health care services using ICT. In IBIMA Conference Proceedings, Milan, Italy (pp. 3541-3552).

13. Kaia Health (2019). Available at: <https:// www.kaiahealth.com>.

14. Mentiplay, B. F., Perraton, L. G., Bower, K. J., Pua, Y. H., McGaw, R., Heywood, S. \& Clark, R. A. (2015). Gait assessment using the Microsoft Xbox One Kinect: Concurrent validity and inter-day reliability of spatiotemporal and kinematic variables, Journal of Biomechanics, 48(10), 2166- 2170.

15. Naeemabadi, M. R., Dinesen, B., Andersen, O. K., Najafi, S. \& Hansen, J. (2018). Evaluating Accuracy and Usability of Microsoft Kinect Sensors and Wearable Sensor for Tele Knee Rehabilitation after Knee Operation. In Proceedings of the 11th International Joint Conference on Biomedical Engineering Systems and Technologies - Volume 1: BIODEVICES, Funchal, Madeira, Portugal (pp. 128-135).

16. Olson, J. S. \& Redkar S. (2018). A Survey of Wearable Sensor Networks in Health and Entertainment, MOJ Applied Bionics and Biomechanics, 2(5), 280-287.

17. O’Reilly, M., Duffin, J., Ward, T. \& Caulfield, B. (2017). Mobile App to Streamline the Development ofWearableSensor-BasedExercise
Biofeedback Systems: System Development and Evaluation, JMIR rehabilitation and assistive technologies, 4(2), e9.

18. Physitrack (2019). The World Leader in Mobile Exercise Prescription. Available at: <https:// www.physitrack. com/>.

19. Radicle (2018). Opportunities in telerehabilitation. Available at: $<$ https:/drive.google.com/ file/d/1hB Wy4tKLZFh2FGElJwoqgDejTuKyOPB/view>.

20. re.flex (2018). Recovery Made Simple. Available at: $<$ http://reflex.help $>$.

21. Reflexion Health (2019). VERA - the Ultimate PT Assistant. Available at: <https:// reflexionhealth.com/vera/>.

22. Shaked, N. A. (2017). Avatars and Virtual Agents - Relationship Interfaces for the Elderly, Healthcare technology letters, 4(3), 83-87.

23. Socker, R. (2018). Wearable technologies. Gadget of the months. Available at: <https:// www.wearable-technologies.com/2018/08/ august-2018/>.

24. SWORD Health (2019). Available at: <https:// swordhealth.com/our-solution/>.

25. Vera-Perez, O. L., Mesejo-Chiong, A., JaumeI-Capó, A. \& Gonzalez-Hidalgo, M. (2014). Automatic parameter configuration: A case study on a rehabilitation oriented human limb tracking algorithm, Studies in Informatics and Control, 23(1), 87-96. DOI: 10.24846/ v23i1y201409

26. Voicu, R. A., Dobre, C., Băjenaru, L. \& Ciobanu, R. I. (2019). Human Physical Activity Recognition Using Smartphone Sensors, Sensors, 19(3), 458.

27. Woolford, K. (2015). Defining Accuracy in the Use of Kinect V2 for Exercise Monitoring. In Proceedings of the 2nd International Workshop on Movement and Computing, British Columbia, Canada (pp. 112-119).

28. Xu, X. \& McGorry, R. W. (2015). The validity of the first and second generation Microsoft Kinect $^{\mathrm{TM}}$ for identifying joint center locations during static postures, Applied Ergonomics, 49, 47-54.

29. Yeung, L. F., Cheng, K. C., Fong, C. H., Lee, W. C. \& Tong, K. Y. (2014). Evaluation of the Microsoft Kinect as a clinical assessment tool of body sway, Gait \& Posture, 40(4), 532-538. 